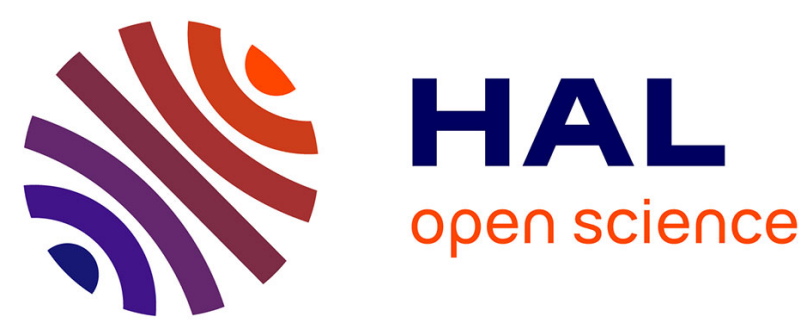

\title{
Identification of artery wall stiffness: in vitro validation and in vivo results of a data assimilation procedure applied to a 3D fluid-structure interaction model
}

Cristobal Bertoglio, David Barber, Nicholas Gaddum, Israel Valverde, Marcel Rutten, Philipp Beerbaum, Philippe Moireau, Rodney Hose, Jean-Frédéric

\author{
Gerbeau
}

\section{To cite this version:}

Cristobal Bertoglio, David Barber, Nicholas Gaddum, Israel Valverde, Marcel Rutten, et al.. Identification of artery wall stiffness: in vitro validation and in vivo results of a data assimilation procedure applied to a 3D fluid-structure interaction model. Journal of Biomechanics, 2014, 47 (5), pp.1027-1034. 10.1016/j.jbiomech.2013.12.029 . hal-00925902v2

\section{HAL Id: hal-00925902 \\ https://hal.inria.fr/hal-00925902v2}

Submitted on 9 Jan 2014

HAL is a multi-disciplinary open access archive for the deposit and dissemination of scientific research documents, whether they are published or not. The documents may come from teaching and research institutions in France or abroad, or from public or private research centers.
L'archive ouverte pluridisciplinaire HAL, est destinée au dépôt et à la diffusion de documents scientifiques de niveau recherche, publiés ou non, émanant des établissements d'enseignement et de recherche français ou étrangers, des laboratoires publics ou privés. 


\title{
Identification of artery wall stiffness: in vitro validation and in vivo results of a data assimilation procedure applied to a 3D fluid-structure interaction model
}

\author{
C. Bertoglio', D. Barber ${ }^{2}$, N. Gaddum ${ }^{3}$, I. Valverde 3 , M. Rutten ${ }^{4}$

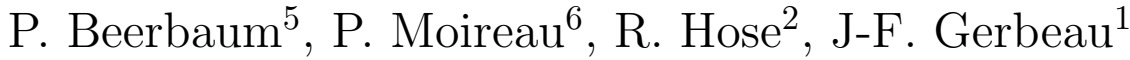 \\ 1 REO team, INRIA Paris-Rocquencourt, France \\ 2 Department of Cardiovascular Sciences, University of Sheffield, UK \\ 3 Division of Imaging Sciences and Biomedical Engineering, King's College London, UK \\ 4 Materials Technology Institute, Technical University of Eindhoven, The Netherlands \\ 5 Clinic for Paediatric Cardiology and Internal Medicine, Hannover Medical School, Germany \\ 6 M3DISIM team, INRIA Saclay Ile-de-France, France
}

Journal of Biomechanics, 2014

\begin{abstract}
We consider the problem of estimating the stiffness of an artery wall using a data assimilation method applied to a 3D fluid-structure interaction (FSI) model. Recalling previous works, we briefly present the FSI model, the data assimilation procedure and the segmentation algorithm. We present then two examples of the procedure using real data. First, we estimate the stiffness distribution of a silicon rubber tube from image data. Second, we present the estimation of aortic wall stiffness from real clinical data.
\end{abstract}

Keywords: fluid-structure interaction; data assimilation; stiffness estimation; medical imaging; experimental validation; coarctation of the aorta

\section{Introduction}

The in vivo estimation of arterial stiffness can provide valuable information about the cardiovascular condition of a patient [21]. In clinical practice, the Pulse Wave Velocity [27] gives an estimation of the average stiffness of a portion of the arterial network by solving the Moens-Korteweg equation, which assumes a linear solid in an infinite cylindrical domain. Other methods include Vascular Elastography, either based on Ultrasound [11, 2, 1] or Magnetic Resonance [32, 33], where the models used for estimating arterial stiffness are again based on simplified models.

We can go further and obtain the local mechanical properties of a vessel if we adopt a data assimilation procedure, based on a three-dimensional fluid-structure interaction model and the distributed kinematic data obtained from dynamic 3D acquisitions (e.g., MRI or $\mathrm{CT}$ ). In this way, stiffness values can be obtained under physiological conditions without requiring invasive pressure measurements.

Recently, [10] proposed an efficient methodology to estimate uncertain parameters (for example Young's modulus or fluid boundary conditions) from measurements of the displacement of the wall in an idealized 3D fluid-structure interaction (FSI) problem. The procedure is based on the Reduced Order Unscented Kalman Filter (ROUKF) [24], which consists of a tractable filtering that allows to solve the estimation problem with a computational effort comparable to one forward simulation. Note that variational (in space and time) approaches 
have been adopted by other authors but for simplified models in [20, 22, 31], and for threedimensional problems in $[12,29]$ but simplifying the minimization problem in order to avoid the resolution of the adjoint equations in time.

Whereas in [10] validations are only based on synthetic data, we consider in the present work the experimental validation of this method using real data in a fluid-structure interaction context. The data come from a laboratory experiment performed on a silicone tube mimicking an aorta. The results show an excellent agreement between the values obtained from data assimilation and those obtained by independent mechanical tests.

We also apply the stiffness estimation methodology to a real clinical case, namely an aorta with a mild coarctation. Hence, this work complements the work of [23] since here we estimate the stiffness parameters and not the boundary conditions.

The rest of the paper is structured as follows. In Section 2 we present the FSI-equations, in Section 3 we summarize the ROUKF algorithm and in Section 4 we overview the image segmentation algorithm. In Section 5 we present the results for stiffness estimation with experimental data and we conclude in Section 6 with the aorta study.

\section{The fluid-structure interaction equations}

Mathematical model We deal with the numerical resolution and the data assimilation of the mechanical interaction between an incompressible fluid and an elastic structure. The fluid is described by the Navier-Stokes equations (NSE), in a moving domain $\Omega^{\mathrm{f}}(t) \subset \mathbb{R}^{d}$, $d=2,3$, in an Arbitrary Lagrangian Eulerian (ALE) formulation, and the structure by the elastodynamic equations in $\Omega^{\mathrm{s}}(t) \subset \mathbb{R}^{d}$. The fluid-structure interface is denoted by $\Sigma=\partial \Omega^{\mathrm{s}} \cap \partial \Omega^{\mathrm{f}}$, and $\partial \Omega^{\mathrm{f}}=\Gamma^{\mathrm{in}} \cup \Gamma_{1} \cup \cdots \cup \Gamma_{n_{0 \mathrm{D}}} \cup \Sigma$ and $\partial \Omega^{\mathrm{s}}=\Gamma^{\mathrm{n}} \cup \Gamma^{\mathrm{d}}$ are given partitions of the fluid and solid boundaries, respectively.

The coupled FSI problem reads as follows: for $t>0$, find the fluid velocity $\boldsymbol{u}_{\mathrm{f}}(t): \Omega^{\mathrm{f}}(t) \rightarrow$ $\mathbb{R}^{d}$, the fluid pressure $p(t): \Omega^{\mathrm{f}}(t) \rightarrow \mathbb{R}$, the structure displacement $\boldsymbol{y}_{\mathrm{s}}(t): \Omega^{\mathrm{s}}(t) \rightarrow \mathbb{R}^{d}$ and structure velocity $\boldsymbol{u}_{\mathrm{s}}(t): \Omega^{\mathrm{s}}(t) \rightarrow \mathbb{R}^{d}$ such that

- Fluid equations:

$$
\left\{\begin{array}{r}
\left.\rho_{\mathrm{f}} \frac{\partial \boldsymbol{u}_{\mathrm{f}}}{\partial t}\right|_{\boldsymbol{\xi}}+\rho_{\mathrm{f}}\left(\boldsymbol{u}_{\mathrm{f}}-\boldsymbol{w}\right) \cdot \boldsymbol{\nabla} \boldsymbol{u}_{\mathrm{f}}-\boldsymbol{\nabla} \cdot \boldsymbol{\sigma}_{\mathrm{f}}\left(\boldsymbol{u}_{\mathrm{f}}, p\right)=\mathbf{0}, \text { in } \Omega^{\mathrm{f}}, \\
\boldsymbol{\nabla} \cdot \boldsymbol{u}_{\mathrm{f}}=0, \text { in } \Omega^{\mathrm{f}}, \\
\boldsymbol{u}_{\mathrm{f}}=\boldsymbol{u}_{\mathrm{in}}, \text { on } \Gamma^{\mathrm{in}}, \\
\boldsymbol{\sigma}_{\mathrm{f}}\left(\boldsymbol{u}_{\mathrm{f}}, p\right) \boldsymbol{n}_{\mathrm{f}}=-P_{i} \boldsymbol{n}_{\mathrm{f}}, \text { on } \Gamma_{i}, i=1, \ldots, n_{0 \mathrm{D}},
\end{array}\right.
$$

with $\boldsymbol{\sigma}_{\mathrm{f}}\left(\boldsymbol{u}_{\mathrm{f}}, p\right)=-p \mathbf{I}+2 \mu_{\mathrm{f}} \boldsymbol{\varepsilon}\left(\boldsymbol{u}_{\mathrm{f}}\right)$, the Cauchy stress tensor of the fluid and $\boldsymbol{\varepsilon}\left(\boldsymbol{u}_{\mathrm{f}}\right)=$ $\left(\boldsymbol{\nabla} \boldsymbol{u}_{\mathrm{f}}+\left(\boldsymbol{\nabla} \boldsymbol{u}_{\mathrm{f}}\right)^{\top}\right) / 2$ denotes the deformation rate tensor, $\mu_{\mathrm{f}}$ the dynamic viscosity, and $\left.\frac{\partial}{\partial t}\right|_{\boldsymbol{\xi}}$ the ALE derivative (see e.g. [13]). In the hemodynamics problems considered in this work, the outlet pressure $P_{i}$ is obtained by solving the differential-algebraic equation, 
known as the Windkessel model [28]

$$
\left\{\begin{array}{l}
P_{i}=\pi_{i}+R_{p, i} Q_{i}, \\
C_{i} \frac{d \pi_{i}}{d t}+\frac{\pi_{i}}{R_{d, i}}=Q_{i}, \quad Q_{i}=\int_{\Gamma_{i}} \boldsymbol{u}_{\mathrm{f}} \cdot \boldsymbol{n}_{\mathrm{f}}
\end{array}\right.
$$

The distal resistance $R_{d, i}$, the proximal resistance $R_{p, i}$ and the capacitance $C_{i}$ are assumed to be given.

- Structure equations:

$$
\left\{\begin{array}{rc}
\partial_{t} \boldsymbol{y}_{\mathrm{s}}=\boldsymbol{u}_{\mathrm{s}}, & \text { in } \Omega^{\mathrm{s}}, \\
\rho_{\mathrm{s}} \partial_{t} \boldsymbol{u}_{\mathrm{s}}-\eta_{\mathrm{s}} \boldsymbol{\nabla} \cdot \boldsymbol{\sigma}_{\mathrm{s}}\left(\boldsymbol{u}_{\mathrm{s}}\right)-\boldsymbol{\nabla} \cdot \boldsymbol{\sigma}_{\mathrm{s}}\left(\boldsymbol{y}_{\mathrm{s}}, \theta\right)=\mathbf{0}, & \text { in } \Omega^{\mathrm{s}}, \\
\boldsymbol{y}_{\mathrm{s}}=\boldsymbol{y}_{\text {in }}, & \text { on } \Gamma^{\mathrm{d}}, \\
\eta_{\mathrm{s}} \boldsymbol{\sigma}_{\mathrm{s}}\left(\boldsymbol{u}_{\mathrm{s}}\right) \boldsymbol{n}_{\mathrm{s}}+\boldsymbol{\sigma}_{\mathrm{s}}\left(\boldsymbol{y}_{\mathrm{s}}\right) \boldsymbol{n}_{\mathrm{s}}=-c_{\Gamma} \boldsymbol{u}_{\mathrm{s}}-k_{\Gamma} \boldsymbol{y}_{\mathrm{s}}, & \text { on } \Gamma^{\mathrm{n}},
\end{array}\right.
$$

where $\boldsymbol{\sigma}_{s}$ is the Cauchy stress tensor of the solid and the vector $\theta \in \mathbb{R}^{\kappa}$ denotes the set of solid constitutive parameters that will be estimated later. In this work, both quantities are related by:

- Considering a linear constitutive relation, namely $\boldsymbol{\sigma}_{s}\left(\boldsymbol{y}_{\mathrm{s}}, \theta\right)=\mathbf{C}(\theta) \boldsymbol{\epsilon}\left(\boldsymbol{y}_{\mathrm{s}}\right)$ with $\boldsymbol{\epsilon}\left(\boldsymbol{y}_{\mathrm{s}}\right)=$ $\left(\boldsymbol{\nabla} \boldsymbol{y}_{\mathrm{s}}+\left(\boldsymbol{\nabla} \boldsymbol{y}_{\mathrm{s}}\right)^{\top}\right) / 2$ and $\mathbf{C}$ the classical elasticity tensor, where the Young's modulus is given by $E=E_{0} \cdot 2^{\theta}, E_{0}>0$, while the Poisson's ratio is fixed.

- Considering a nonlinear, hyperelastic, neo-hookean material model, such that the elastic energy density function is given by $\mathcal{W}=c_{0} \cdot 2^{\theta} \cdot\left(I_{1}-3\right), c_{0}>0$ and $I_{1}$ the first invariant of the right Cauchy-Green strain tensor.

Moreover, the parameters $c_{\Gamma}$ and $k_{\Gamma}$ model in a simple way the external tissue effect on the vessel of interest $[26,23]$ and $\eta_{\mathrm{s}}$ is a viscoelastic coefficient.

- Coupling conditions:

$$
\left\{\begin{array}{r}
\boldsymbol{y}_{\mathrm{f}}=\operatorname{Ext}_{\Sigma(0)}^{\mathrm{f}}\left(\boldsymbol{y}_{\left.\mathrm{s}\right|_{\Sigma}}\right), \boldsymbol{w}=\partial_{t} \boldsymbol{y}_{\mathrm{f}}, \Omega^{\mathrm{f}}(t)=\left(I_{\Omega^{\mathrm{f}}}+\boldsymbol{y}_{\mathrm{f}}(t)\right)\left(\Omega^{\mathrm{f}}\right), \\
\boldsymbol{u}_{\mathrm{f}}=\boldsymbol{u}_{\mathrm{s}}, \text { on } \Sigma, \\
\eta_{\mathrm{s}} \boldsymbol{\sigma}_{\mathrm{s}}\left(\boldsymbol{u}_{\mathrm{s}}\right) \boldsymbol{n}_{\mathrm{s}}+\boldsymbol{\sigma}_{\mathrm{s}}\left(\boldsymbol{y}_{\mathrm{s}}\right) \boldsymbol{n}_{\mathrm{s}}+\boldsymbol{\sigma}_{\mathrm{f}}\left(\boldsymbol{u}_{\mathrm{f}}, p\right) \boldsymbol{n}_{\mathrm{f}}=\mathbf{0}, \text { on } \Sigma,
\end{array}\right.
$$

where $\operatorname{Ext}_{\Sigma}^{\mathrm{f}}$ is an extension operator from $\Sigma$ to $\Omega^{\mathrm{f}}$.

FSI numerical algorithm The spatial discretization is performed with a first order stabilized finite element. For the time marching, we use a semi-implicit partitioned FSI-algorithm $[14,15]$ with a first order Chorin-Temam projection method in the fluid, and a Newmark scheme in the solid. The coupling between the solid and the fluid is explicit in the ALEadvection-diffusion step and implicit for the pressure projection step and the Windkessel [8]. 


\section{The data assimilation procedure}

In this section we summarize the data assimilation procedure based on [24] and already studied in [10] for idealized (but realistic) three-dimensional arterial FSI problems.

Inverse problem statement Assume that we can write the fully discrete FSI-problem as

$$
X_{n+1}=\mathcal{A}_{n}\left(X_{n}, \theta\right), n \geq 0,
$$

with $X_{n}=\left(\boldsymbol{u}_{\mathrm{f}}^{n}, \boldsymbol{y}_{\mathrm{s}}^{n}, \boldsymbol{u}_{\mathrm{s}}^{n}\right) \in \mathbb{R}^{q}$ the discrete dynamical state, $\theta \in \mathbb{R}^{\kappa}$ the physical parameters, and $X_{0}$ the given inititial condition. Suppose that $\theta$ and $X_{0}$ are uncertain, and that we have (noised) measurements of real physical system $Z_{n} \in \mathbb{R}^{z}$ related to the numerical state by the observation error $\Gamma_{n}\left(Z_{n}, X_{n}\right)=\zeta_{n}$, with $\zeta_{n} \in \mathbb{R}^{m}$ the noise. Data assimilation consists in reducing the uncertainties of a model by minimizing a cost function like (see, e.g., [3]):

$$
J\left(X_{0}, \theta_{0}\right)=\sum_{n=0}^{N}\left\|\Gamma_{n}\left(Z_{n}, X_{n}\right)\right\|_{W_{n}^{-1}}^{2}+\left\|\theta_{0}-\hat{\theta}_{0}^{+}\right\|_{\left(P_{0}^{\theta}\right)^{-1}}^{2}+\left\|X_{0}-\hat{X}_{0}^{+}\right\|_{\left(P_{0}^{X}\right)^{-1}}^{2},
$$

with $X_{n}$ satisfying (2). In this expression, $\hat{X}_{0}$ and $\hat{\theta}_{0}$ are given a priori values and $\|\cdot\|_{W^{-1}}$, $\|\cdot\|_{\left(P_{0}^{X}\right)^{-1}}$ and $\|\cdot\|_{\left(P_{0}^{\theta}\right)^{-1}}$ denote some matrix norms used to give a different weight to the different terms.

This minimization problem can be addressed by many methods that are classically divided in two groups: the variational and the sequential approaches. Variational approaches minimize this cost function by an optimization algorithm - usually a gradient-based computed from the solution of an adjoint model - and require numerous computations of the forward - and possibly the adjoint - problem, see e.g. [22]. Here, we follow a sequential, or filtering, approach which modifies the forward dynamics (2) with a correction term proportional to the observation error. More precisely, we adopt the Reduced-Order Unscented Kalman Filter (ROUKF) [24], inspired from [18, 19]. It does not require any tangent operator and allows one to run the estimation with a computational cost of the same order of the forward problem since it is highly parallelizable. Moreover, it requires only superficial modifications to the existing solvers. Its main assumption consists of neglecting the uncertainty in $X_{0}$. Hence $P_{0}^{X} \rightarrow \infty$ and a reduced-order form of the Kalman filter can be obtained. Note that it is also possible to take into account the uncertainties in the initial condition in the same framework with an additive nudging based correction on the dynamics as introduced and analyzed in [25, 9] and illustrated in [23].

In fact, dealing with uncertainties in the initial condition is a difficult task, which is discussed in detail in [9] for the FSI case.

The ROUKF algorithm Consider the matrix of simplex sigma-points computed recursively as

$$
\left[I_{1}^{(*)}\right]=\left(-\frac{1}{\sqrt{2 \alpha}}, \frac{1}{\sqrt{2 \alpha}}\right), \alpha=\frac{1}{\kappa+1}
$$


and for $2 \leq d \leq \kappa$

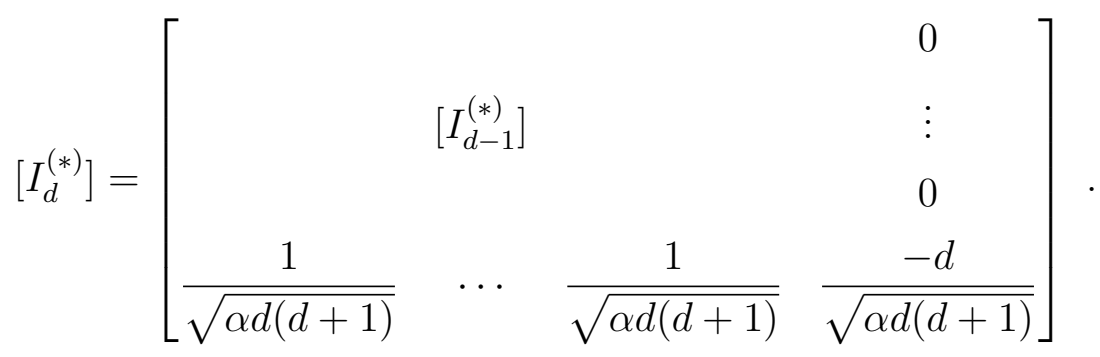

$[30,17]$. Then, for given values of $\hat{X}_{0}^{+}, \hat{\theta}_{0}^{+}$and $P_{0}^{\theta}$, perform

\section{- Initialization:}

$$
L_{0}^{\theta}=\mathbb{I}, \quad L_{0}^{X}=0, \quad U_{0}=\left(P_{0}^{\theta}\right)^{-1},
$$

with $L_{0}^{\theta}$ and $L_{0}^{X}$ the initial sensitivities for the parameters and initial condition, respectively. Note that this assumes that the uncertainty at the initial time step is present only in the parameters and not in the initial condition, see [24] for details. Then for $n=0, \ldots, N-1$ perform:

\section{- Sampling:}

$$
\left\{\begin{array}{l}
C_{n}=\sqrt{U_{n}^{-1}} \quad(\text { Cholesky factorization }) \\
\hat{X}_{n}^{(i)+}=\hat{X}_{n}^{+}+L_{n}^{X} C_{n}^{\top} I_{\kappa}^{(i)}, \quad 1 \leq i \leq \kappa+1, \\
\hat{\theta}_{n}^{(i)+}=\hat{\theta}_{n}^{+}+L_{n}^{\theta} C_{n}^{\top} I_{\kappa}^{(i)}, \quad 1 \leq i \leq \kappa+1,
\end{array}\right.
$$

where $\hat{X}_{n}^{(i)+}$ and $\hat{\theta}_{n}^{(i)+}$ are classically called particles, and $(\cdot)^{(i)}$ denotes the $i$ th-column of the matrix $\left[(\cdot)^{(*)}\right]$.

\section{- Propagation:}

$$
\left\{\begin{array}{l}
\left(\hat{X}_{n+1}^{(i)-}, \hat{\theta}_{n+1}^{(i)-}\right)=\mathcal{A}_{n+1}\left(\hat{X}_{n}^{(i)+}, \hat{\theta}_{n}^{(i)+}\right), \quad i=1, \ldots, \kappa+1, \\
\hat{\Gamma}_{n+1}^{(i)}=\Gamma\left(Z_{n+1}, \hat{X}_{n+1}^{(i)-}\right), \quad i=1, \ldots, \kappa+1,
\end{array}\right.
$$

which can be performed independently for each $i$, leading to the intrinsical parallel feature of this algorithm.

\section{- Prediction:}

$$
\left\{\begin{array}{l}
\hat{X}_{n+1}^{-}=\alpha \sum_{i=1}^{\kappa+1} \hat{X}_{n+1}^{(i)-} \\
\hat{\theta}_{n+1}^{-}=\alpha \sum_{i=1}^{\kappa+1} \hat{\theta}_{n+1}^{(i)-} \\
\hat{\Gamma}_{n+1}^{-}=\alpha \sum_{i=1}^{\kappa+1} \hat{\Gamma}_{n+1}^{(i)-}
\end{array}\right.
$$




\section{- Correction:}

$$
\left\{\begin{array}{l}
L_{n+1}^{X}=\alpha\left[\hat{X}_{n+1}^{(*)}\right]\left[I_{p}^{(*)}\right]^{\top} \\
L_{n+1}^{\theta}=\alpha\left[\hat{\theta}_{n+1}^{(*)}\right]\left[I_{p}^{(*)}\right]^{\top} \\
L_{n+1}^{\Gamma}=\alpha\left[\Gamma_{n+1}^{(*)}\right]\left[I_{p}^{(*)}\right]^{\top} \\
U_{n+1}^{\top}=P_{\alpha}+\left(L_{n+1}^{\Gamma}\right)^{\top} W_{n+1}^{-1} L_{n+1}^{\Gamma} \\
\hat{X}_{n+1}^{+}=\hat{X}_{n+1}^{-}-L_{n+1}^{X} U_{n+1}^{-1}\left(L_{n+1}^{\Gamma}\right)^{\top} W_{n+1}^{-1} \hat{\Gamma}_{n+1}^{-} \\
\hat{\theta}_{n+1}^{+}=\hat{\theta}_{n+1}^{-}-L_{n+1}^{\theta} U_{n+1}^{-1}\left(L_{n+1}^{\Gamma}\right)^{\top} W_{n+1}^{-1} \hat{\Gamma}_{n+1}^{-} .
\end{array}\right.
$$

Here we choose $W_{n+1}^{-1}=\gamma M_{\Sigma}$, with $M_{\Sigma} \in \mathbb{R}^{m \times m}$ the $L^{2}$-mass matrix on the fluidstructure interface and $\gamma>0$.

Observation operator As a meaningful observation we rely here on the available segmentations of medical images usually given as a set of surfaces, here the fluid-structure interface at each acquisition time. Hence, we quantify an observation error by computing the (signed) distance field between the segmented surfaces and the deformed fluid-structure interface [25].

\section{Image registration based segmentation}

The data assimilation process requires estimates of the displacements of points on the vessel wall at intervals over the cardiac cycle, typically measured from segmentations of dynamic image data. In this work, the segmentation is performed by registration of one reference image to each frame (target image) of the dynamic images using the Sheffield Image Registration Toolkit (ShIRT). There follows a brief summary of the algorithm and implementation: for more details see $[4,5]$.

Image registration algorithm The registration process seeks to determine the displacement field that brings the reference image into alignment with the target image, assuming that the intensities of corresponding points on the two images are related by

$$
f(\boldsymbol{x}+\boldsymbol{u})=m(\boldsymbol{x})
$$

with $f$ and $m$ the intensities of the fixed and moving images, respectively, and $\boldsymbol{x}=[x, y, z]$ the cartesian spatial coordinates and $\boldsymbol{u}=[u, v, w]$ the displacement field.

If the objects in the images have the same topology and, crucially, the same ranges of intensity then it is possible in principle to find a displacement field $u$ that satisfies Equation (4) exactly. Otherwise it is satisfied with minimum error described by a cost function. In fact small variation in image intensity ranges, including gradients across the image that are commonly encountered in some modalities, can be accommodated by adding an additional image dimension, $s$, that represents the intensity of the image. Thus $f(\boldsymbol{x})$ and $m(\boldsymbol{x})$ are then transformed to $\hat{f}(\hat{\boldsymbol{x}})$ and $\hat{m}(\hat{\boldsymbol{x}})$, respectively, with $\hat{\boldsymbol{x}}=[x, y, z, s]$, and $\hat{f}, \hat{m}$ taken binary values, with the height of each column in the $s$ direction equal to the intensity of the image at that point. The displacement $\boldsymbol{u}$ is redefined now including a term in the $s$ direction, i.e.,

$$
\hat{f}(\hat{\boldsymbol{x}}+\hat{\boldsymbol{u}})=\hat{m}(\hat{\boldsymbol{x}}), \hat{\boldsymbol{u}}=\left[u, v, w, u_{s}\right] .
$$


For small values of the displacements, the relationship between the intensities of the two images at the common coordinate $\boldsymbol{x}$ is given by

$$
\hat{m}(\hat{\boldsymbol{x}})=\hat{f}(\hat{\boldsymbol{x}})+\left.\hat{\boldsymbol{u}} \cdot\left(\boldsymbol{\nabla}_{\hat{\boldsymbol{x}}} \hat{f}\right)\right|_{\hat{\boldsymbol{x}}}
$$

Note that the registration problem can also be formulated in terms of the same displacements applied to the image $m$, i.e.

$$
\hat{f}(\hat{\boldsymbol{x}})=\hat{m}(\hat{\boldsymbol{x}})-\left.\hat{\boldsymbol{u}} \cdot\left(\boldsymbol{\nabla}_{\hat{\boldsymbol{x}}} \hat{m}\right)\right|_{\hat{\boldsymbol{x}}} .
$$

Combining both Equations (6) and (7) we can define the following residual for the registration

$$
\mathcal{R}(\hat{\boldsymbol{x}}) \stackrel{\text { def }}{=} \hat{m}(\hat{\boldsymbol{x}})-\hat{f}(\hat{\boldsymbol{x}})-\left.\frac{\hat{\boldsymbol{u}}}{2} \cdot\left(\boldsymbol{\nabla}_{\hat{\boldsymbol{x}}}(\hat{m}+\hat{f})\right)\right|_{\hat{\boldsymbol{x}}}
$$

which in our experience improves the performance of the registration compared with considering the residuals only based on Equations (6) or (7).

The registration algorithm solves then a sequence of regularized linear least-squares problems based on a cost function that evaluates the discrete residual in Equation (8) after registration, namely, for $p>1$ solve

$$
\underset{U_{p}}{\operatorname{argmin}}\left(\frac{1}{2}\left\|(M-F)-[T] U_{p}\right\|^{2}+\frac{\lambda}{2}\left\|[\Delta] \sum_{q=1}^{p-1} U_{q}\right\|^{2}\right)
$$

with $U_{p}$ the degrees-of-freedom of the trilinear interpolation of $\hat{\boldsymbol{u}},[\Delta]$ corresponds to the discrete Laplacian operator included as the regularisation term (with $\lambda>0$ the corresponding weighting factor), and the matrix $[T]$ arises from the discretisation of the gradients in Equation (8).

The process is assumed to be converged when the change of $U_{p}$ over an iteration is less than a pre-determined value. At this stage the grid resolution is increased and the operation starts again, producing a converged solution at the next level of grid refinement. In principle this can continue until the grid on which the displacement is defined is equal to the voxel resolution of the images, but in practice there is little benefit in reducing the grid below $2 \times 2 \times 2$ voxels.

Registration-based segmentation of the MR-data For the elastic tube phantom reported in Section 5 , the reference image is determined by registering each frame in the time series back to the first frame and then averaging the result. The mesh points that will be passed to the data assimilation step are determined by registering an image of a hollow cylinder on which a mesh is defined to the reference image and then propagating the interpolated displacements from the registration of the time series images. The initial segmentation of the human aorta reported in Section 6 is performed by operation on a 3D contrast-enhanced MR image, using an extension of ShIRT that performs a series of registrations to grow the segmentation from a regional seed. This is preferred to direct segmentation of one frame of the dynamic image because the image quality is better. A triangular surface mesh is defined on the surface of the resulting lumen, and this mesh is mapped onto the dynamic image at one (diastolic) frame, 
again by registration, with trilinear interpolation of the registration field. This reference frame is registered to each frame of the dynamic image in turn, and the displacements of the nodes of the mesh are again determined by trilinear interpolation. Note that despite the non-physical assumptions in the registration procedure leading to potential errors in the displacements computed at this stage, the surfaces generated by the segmentation are accurate and therefore meaningful as an input in the data assimilation procedure presented in Section 3 (see also [23]).

\section{In vitro validation: a silicon rubber aortic phantom}

Experimental setting The experimental setup is described in Figure 1, and consists of a MR-compatible emulator of the systemic circulation. The fluid pump corresponds to a linear servo actuator (ETB-32, Parker Hannifin) and piston assembly, located outside the 5 Gauss line coupled to the ventricle piston via a $2 \mathrm{~m}$ rigid boom. The piston ejects water through a tri-leaflet polyurethane valve, (Hemolab, Eindhoven, The Netherlands), and into a $450 \mathrm{~mm}$ length, $18.5 \mathrm{~mm}$ diameter and $1 \mathrm{~mm}$ thickness silicone tube, (TU Eindhoven, The Netherlands), with an elastic strap distally to make it stiffer there. Afterload is maintained by a mechanical Windkessel model at the distal end of the tube comprising fluid resistance, then compliance, then another resistance. After that the working fluid returns to the pump through a venous reservoir and an additional valve. At the centre of the aorta length, a rubber collar was prepared and applied to simulate a local coarctation. The collar was held in place by a spot of glue at its proximal end.

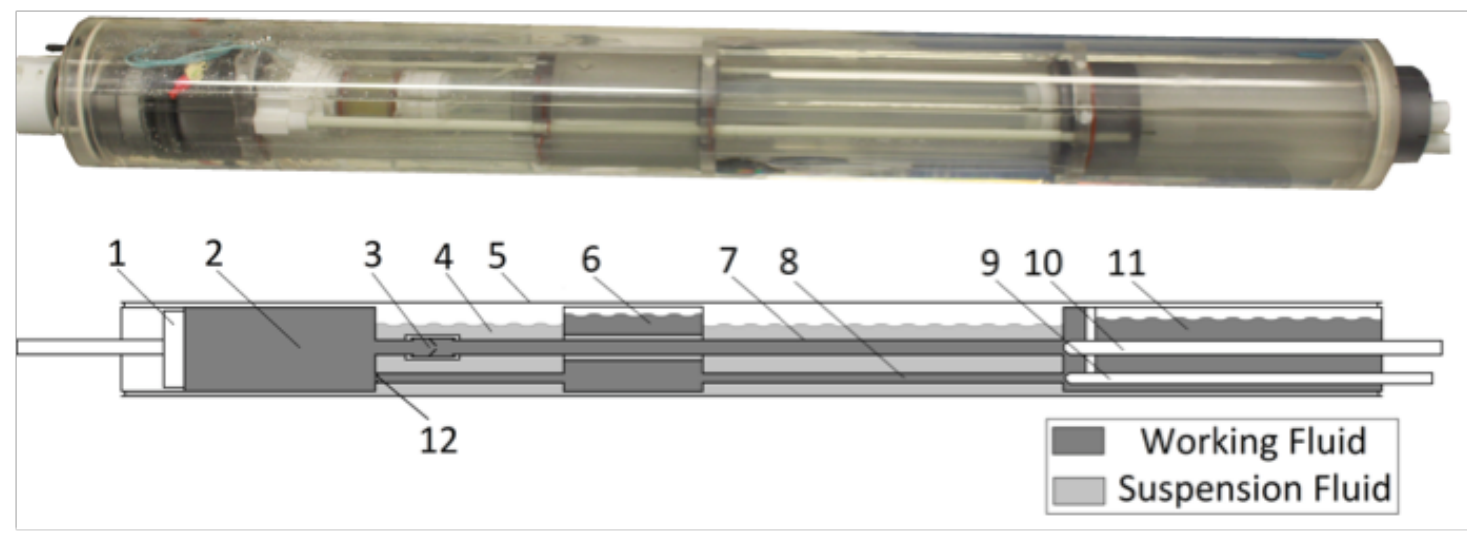

Figure 1: MRI compatible mock ventricle and aorta pulsatile phantom, where; 1 , reciprocating ventricle piston; 2, ventricle and working fluid; 3 , arterial valve; 4, suspension fluid; 5, outer cylindrical case; 6 , venous compliance chamber; 7 , silicone aorta; 8 , venous channel; 9 , venous resistance screw; 10 , arterial resistance screw and arterial catheter access; 11, arterial compliance chamber; 12 , venous valve.

Measured data Pressure measurements were taken at 13 locations between the venous and arterial compliance chambers with a measurement spacing of $20 \mathrm{~mm}$ using a pressure wire, (Certus, St. Jude Medical Systems, Uppsala, Sweden) at a temporal resolution of $1000 \mathrm{~Hz}$. 
The diastolic values for the pressure vary along the tube between 26 and $29 \mathrm{~mm} \mathrm{Hg}$, and the systolic between 105 and $110 \mathrm{mmHg} 0.3 \mathrm{~s}$ later.

Then, MR-acquisitions were performed on a 3T scanner, (Philips Achieva, Philips Healthcare, Best, The Netherlands). Geometry imaging was captured in a balanced turbo field echo (B-TFE) cine scan with a temporal resolution of $0.01 \mathrm{~s}$ and 13 slices, the same of the pressure measurements, were taken with an excitation slice thickness of $8 \mathrm{~mm}$, and a pixel size of $1.8 \times 2.5 \mathrm{~mm}$. Image segmentation was then carried out using the methodology described in Section 4.

A non-destructive mechanical test was then performed consisting in placing the tube (without the strap) vertically, filling it with water, and then injecting an additional controlled water volume while measuring the pressure at the bottom, see Figure 2. A pressure dependent Young's modulus was then constructued using basic linear solid mechanics theory as

$$
E(p) \approx \frac{(2-\nu) V^{\frac{3}{2}} \delta p}{\sqrt{L \pi} \delta V h}
$$

with $\nu$ the Poisson's ratio, $h$ the tube thickness, $L$ the tube length and $\delta p$ and $\delta V$ the measured volume and pressure increments, respectively.

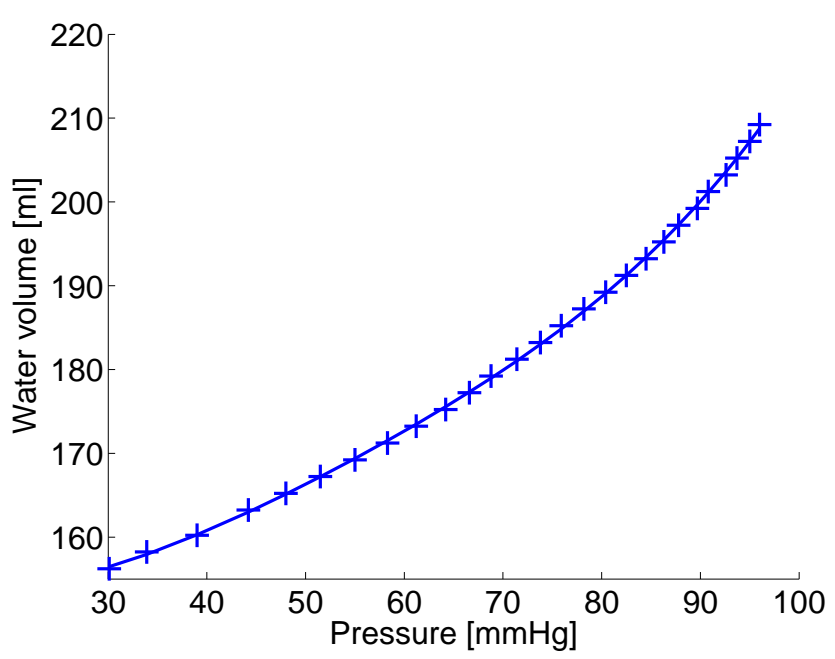

Figure 2: Pressure $P_{i}$ and volume $V_{i}, i=1, \ldots, 27$, acquired from the mechanical test. The raw data is plot with + and the filtered data (4th order interpolation of Volume-Pressure data) with continues lines.

The FSI model The geometry used for the mesh construction (and also initial condition) was chosen as the segmented surfaces at time $0.19 \mathrm{~s}$, where the pressure varies between 44 and $50 \mathrm{mmHg}$ along the tube. The surface was then remeshed using 3-matic (Materialise, Leuven, Belgium), and the volume was closed with additional surfaces at the outlet and the inlet. Then, using Gmsh [16], the surface was extruded outwards the cylinder in two tetrahedral layers with total thickness of $1 \mathrm{~mm}$. Finally both solid's and fluid's volume meshes were constructed with 135840 and 255023 tetrahedra, respectively, resulting from 
setting the mesh size in the mesh generation processs between $1 \mathrm{~mm}$ and $1.5 \mathrm{~mm}$. The boundary conditions for the fluid were directly taken from the pressure measurements at inlet and outlet. In the experiment, the real tube was radially and axially constrained at the physical boundaries. However, since the limits of the computational domain are away from the constrained boundaries, in the FSI-model we only fixed both inlet and outlet axially. Hence, in practice we neglected the radial stiffness of the parts of the tube not considered in the analysis. The physical parameters of Model (1) were chosen as: $c_{\Gamma}=10^{4}, k_{\Gamma}=0$, $\mu=0.035, \rho_{\mathrm{f}}=1, \rho_{\mathrm{s}}=1.2, \nu=0.45, \eta_{\mathrm{s}}=0$. Since the initial pressure field is not zero, the loads received by the solid are corrected by the initial one, so that the system starts at rest.

Estimation results We subdivide the solid volume in 10 regions and estimate simultaneously the Young's modulus for each region. As it can be seen in Figure 3, the estimation algorithm succesfully detects the stiffer regions (first and second, which correspond to the actual placement of the strap), while in the regions where the strap is not presented the stiffness values are close to the one derived from the mechanical test. Specifically, the estimated stiffness in regions 3 and 4 match perfectly the experimental value, while the rest gradually increases when approaching the end of the tube. This could be explained by the fact that we did not include any radial stiffness in order to represent the neglected part of the tube in the computational model.

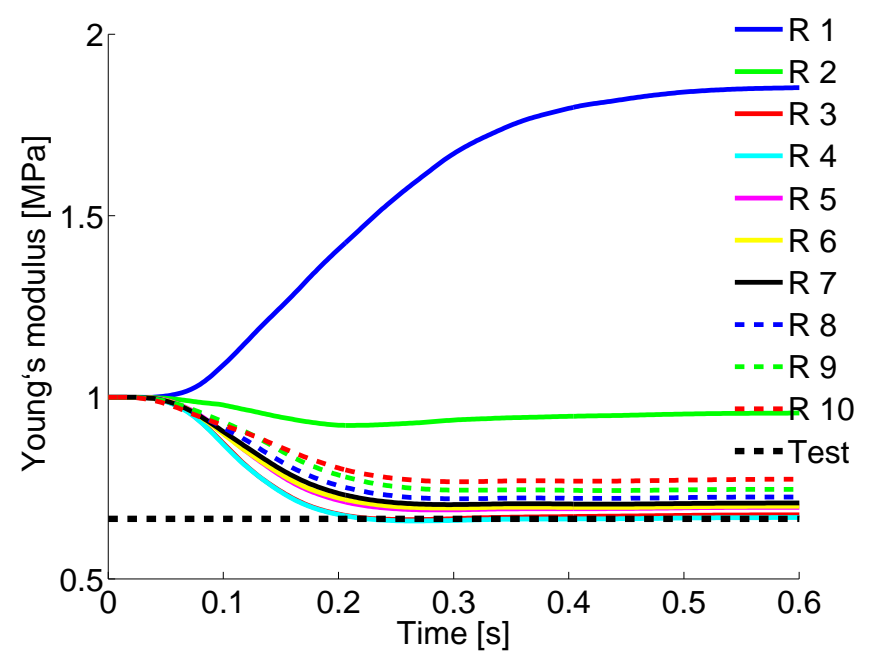

Figure 3: Estimation results for the 10 regions, with $\gamma=10$ and reference value obtained from the mechanical test and the formula (9) with $p=47 \mathrm{mmHg}$.

\section{In vivo results: an aorta with mild coarctation}

Clinical data The available clinical data corresponds to a 19 year old male subject with a mild (repaired) coarctation of the descending aorta, see Figure 4. A static 3D MR-image 
was obtained with a 10-seconds untriggered acquisition and a gadolinium contrast agent with voxel size is $1.36 \times 1.36 \times 1.8 \mathrm{~mm}$ and then segmented using with a registration based segment growing algorithm [6] (see also Section 4). A 4D-SSFP sequence was acquired (25 time frames) along the cardiac cycle of $1.324 s$ were acquired with a voxel size of $1.29 \times 1.29 \times$ $2.4 \mathrm{~mm}$. Consequently, the 25 surfaces were segmented with the template mesh obtained from the 3D-Gd, see Figure 5. Four fixed-in-space slices of in-plane velocity phase contrast MRimages Also invasive pressures were measured at the ascending, descending, and abdominal aorta.

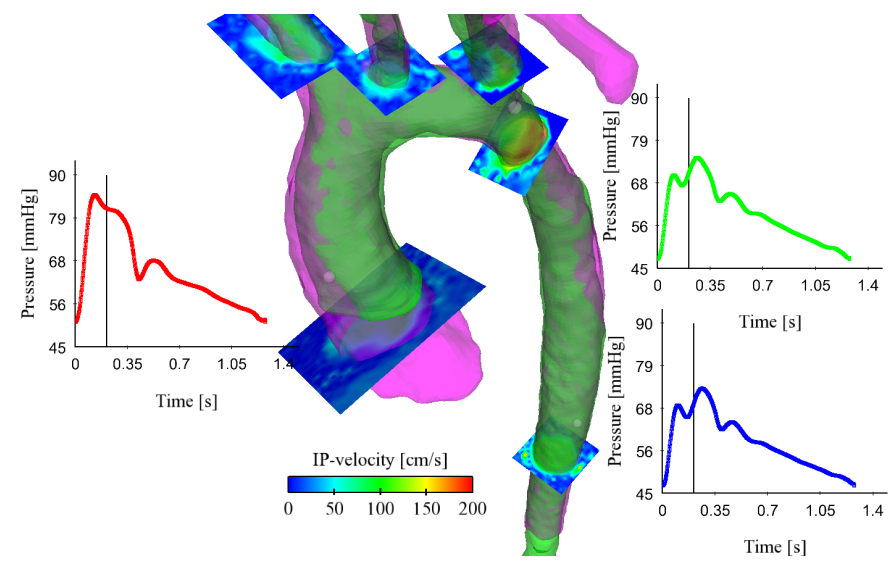

Figure 4: Measurements available for the study case 10 of the euHeart database. Static segmentation (pink geometry); dynamic segmentation (green geometry); invasive pressure measurements at three locations: ascending (red), descending (green) and abdominal (blue) aorta; and In-Plane velocity measurements.

The FSI model For the mesh construction, we proceed concordingly as for the phantom, starting from the first segmented surface from the 4D-SSFP secquence, obtaining both solid

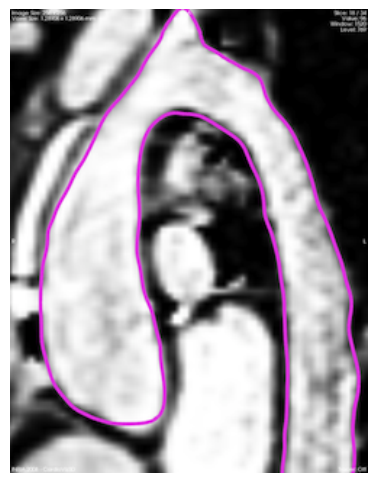

(a) $t=0 \mathrm{~s}$

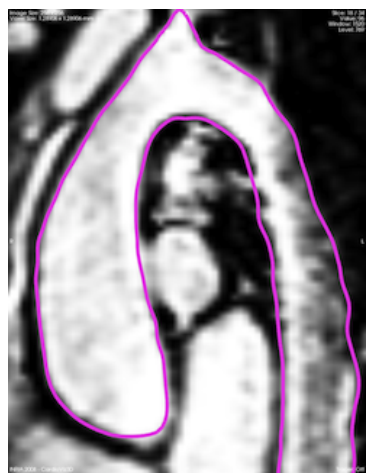

(b) $t=0.110 \mathrm{~s}$

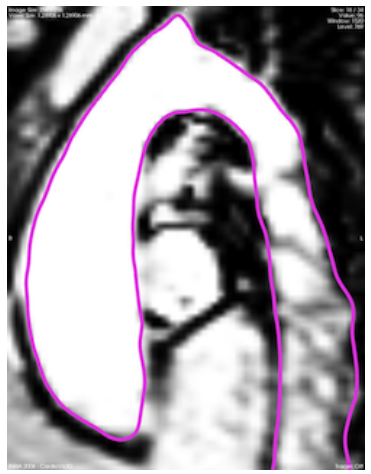

(c) $t=0.221 \mathrm{~s}$

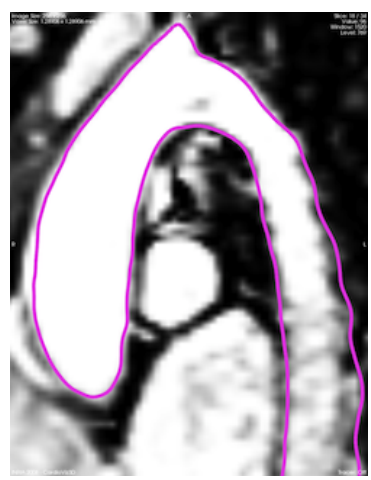

(d) $t=0.386 \mathrm{~s}$

Figure 5: Four sample time frames and the segmented surfaces (in magenta) for a representative slice of the $4 \mathrm{D}$-SSFP sequence. Notice the changes of contrast of the images due to the changes in blood velocity. 
and fluid volume meshes with 103406 and 138648 tetrahedra, respectively, resulting from setting the mesh size in the mesh generator between $0.5 \mathrm{~mm}$ and $1.5 \mathrm{~mm}$, where an arterial wall thickness of $2 \mathrm{~mm}$ was assumed.

The physical parameters were chosen as: $\mu=0.035, \rho_{\mathrm{f}}=1, \rho_{\mathrm{s}}=1.2$ and $\eta_{\mathrm{s}}=10^{5}$. The solid volume was divided into 5 regions (see Figure 6-left) where a Mooney-Rivlin constitutive model with parameters $c_{1}^{j}=3 \cdot 10^{5} \cdot 2^{\theta^{j}}, c_{2}^{j}=10^{4}$ and $\kappa^{j}=10^{8}$ for each region $j=1, \ldots, 5$. The parameters $\theta_{j}$ will be estimated later. The factor $3 \cdot 10^{5}$ was chosen so that the overall vessel deformation is of the order of the one observed in the images with $\theta_{j}=0$. The solid boundary conditions parameters where chosen as: $k_{\Gamma}=0$ for the supra-aortic branches, and $k_{\Gamma}=10^{5}$ for the abdominal outlet, while $c_{\Gamma}=10^{4}$ everywhere on the outer surface. Inspired by [26], we extract a displacement field from the segmented surfaces and impose it at the model's inlet for taking the heart motion into account, see [7] for details.

A plane velocity profile in the inlet at the level of the ascending aorta was imposed, with amplitude obtained by the flow from the IP-velocity data (20 lit/min at $0.15 \mathrm{~s}$ ). The Windkessel models were calibrated manually in order to approach the measured fluxes and pressures, see [7] for details. The load sent from fluid to structure was again corrected by the initial one.

Estimation results The estimation results are presented in Figure 6. We see that the parameters adapt in time in order for the simulation to pursue the MR images. In fact, we present in Figure 7 some time frames of the MR-images, segmentations and models with the initial and calibrated parameters, showing that the model with estimated parameters is closer to the segmented surfaces. However, the results do not converge as in the in vitro validation. This is most probably due to modelling errors, since for example external tissue supports are not taken into account in this case. Another likely cause lies in the low signal-to-noise ratio (SNR) (see Figure 5), in particular in the coarctation region. Hence, the estimated stiffness parameters evolve and oscillate in time in order to compensate for these modelling and data errors. Despite this lack of convergence, the parameter estimation process shows reasonable robustness, as verified with multiple estimation cycles, see in Figure 8 where we relaunch the estimation starting from the previously estimated parameters - but keeping the same initial unitary covariance matrix. We remark, indeed, that the initial and final values get closer after each estimation run and the oscillations stabilize. Hence, we prove here that our method is of practical use for complex clinical data and simulation objectives, and represents a powerful tool to identify patient-specific parameters and to assess necessary improvements for the model or the data processing.

\section{Conclusions}

We performed an in vitro validation of the parameter estimation algorithm for coupled fluid-structure systems presented in [10]. For this purpose, we considered a silicon rubber tube emulating the aorta, connected with a mechanical model of the main components of the cardiovascular system. We constructed a FSI computational model of the cylinder based on the data segmented from dynamic MR-images and we used pressure measurements for the fluid boundary conditions. We estimated the Young's modulus for a linear solid. We verified 

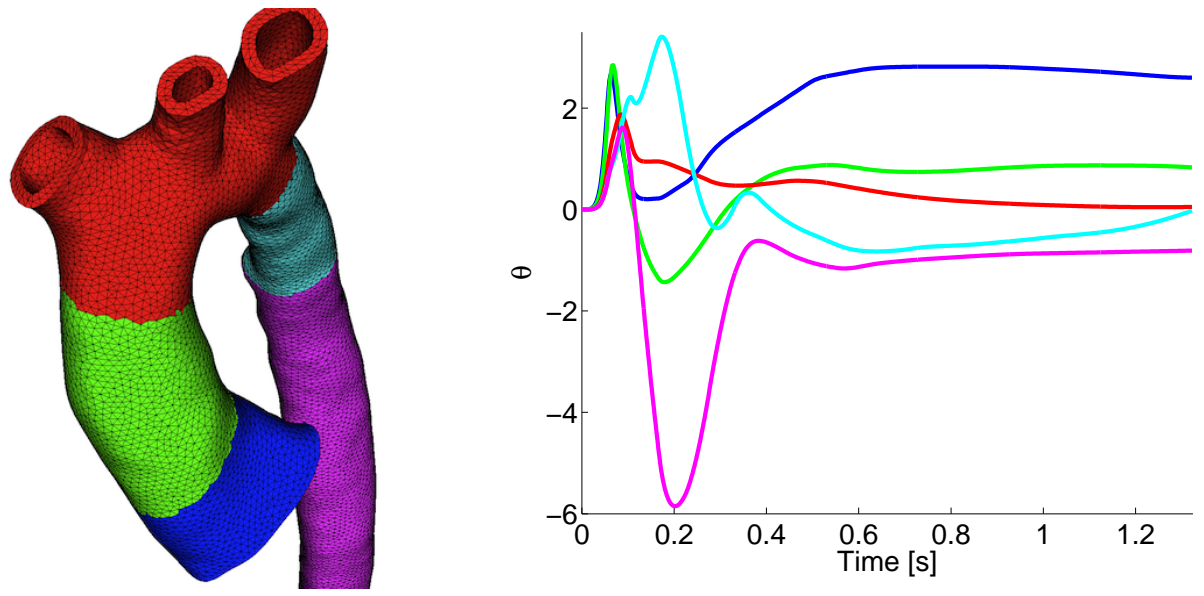

Figure 6: Estimation results for the constitutive parameters $\theta^{j}, j=1, \ldots, 5$, using the segmented surfaces. Left: geometry of the model's aorta and subdivisions of the solid domain. Right: mean estimated values with $\gamma=10^{3}$ and $P_{0}^{\theta}=\mathbb{I}$.

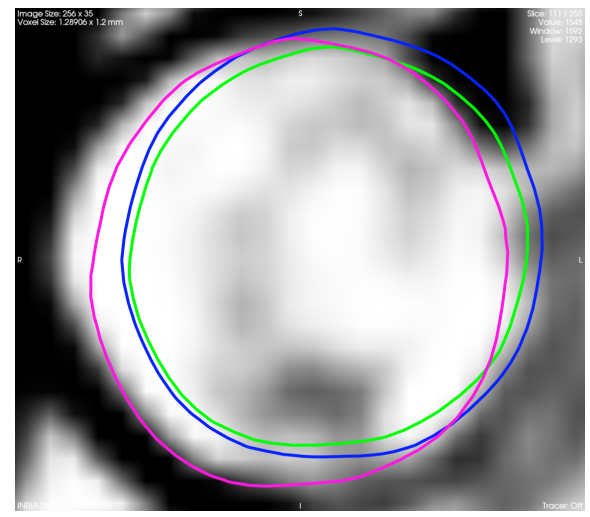

(a) $t=0.21 \mathrm{~s}$

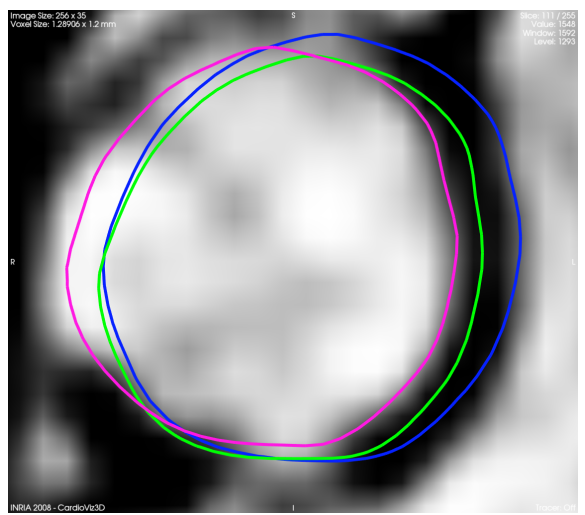

(b) $t=0.53 \mathrm{~s}$

Figure 7: Comparison for some time instants between image, segmented surface (magenta), and FSI-model with initial (blue) and optimal (green) parameters. The view corresponds to a foot-head cut of the ascending aorta at the level of the pulmonary trunk. 

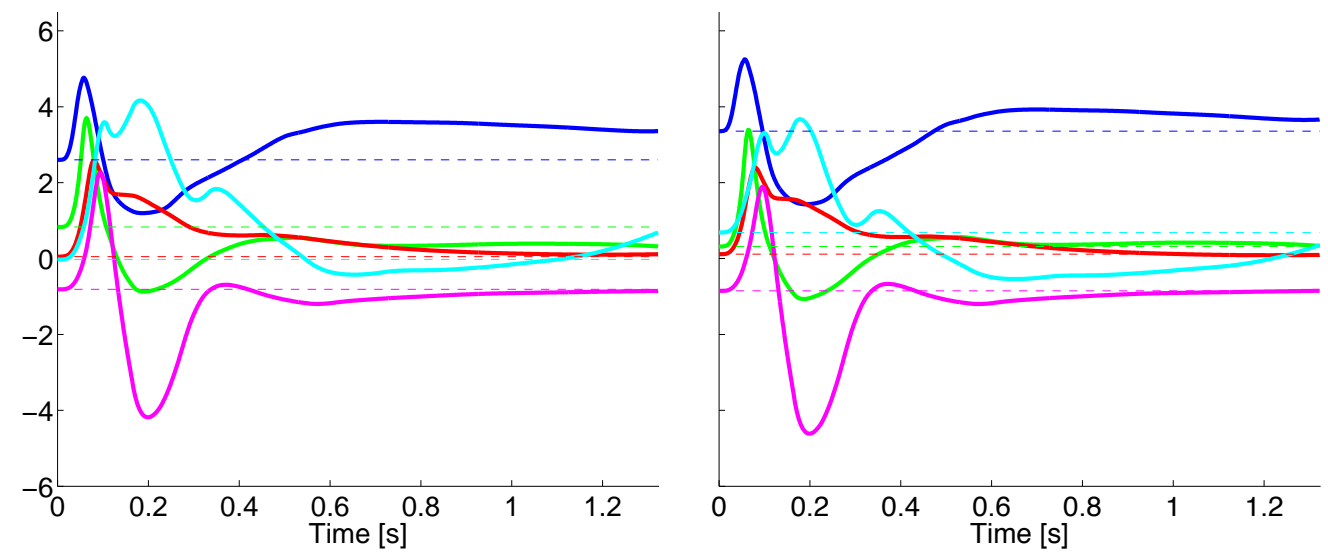

Figure 8: Estimation results for the constitutive parameters $\theta^{j}, j=1, \ldots, 5$, using the segmented surfaces starting from previously estimated values. The dashed values correspond to the initial guess. Left: Second run. Right: Third run.

one of the estimated parameters from data coming from an independent non-destructive mechanical test. In addition, we show that we can straightforwardly use the algorithm in a real clinical case, namely for the estimation of the Mooney-Rivlin constitutive parameters in an aorta with repaired coarctation from the 4D-SSFP sequence, obtaining meaningful results for the estimated parameter values, in the sense that the model with estimated parameters follows closer the segmented surfaces. However, in order to fully exploit the potential of the parameter estimation process, we should also improve both modelling and data acquisition procedures, including the sliding-contact of the aorta with surrounding tissues, more realistic constitutive models and prestress, together with improvements in the quality of the acquired geometry data and fluid field measurements for a better pressure field estimation.

In conclusion, this work illustrates that the combination of automatic image segmentation tools, detailed fluid-structure models, efficient numerical algorithms for their simulation and robust data assimilation procedures provide a powerful toolchain for the in vivo analysis of arterial tissue properties.

\section{Conflict of interests statement}

The authors of this manuscript have no conflict of interest with the presented work.

\section{Acknowledgements}

The research leading to these results has received funding from the European Community's Seventh Framework Programme (FP7/2007-2013) under grant agreement 224495 (euHeart project). We also thank sincerely to Cristina Staicu (U. Sheffield) for assistance in the data processing, and to Miguel A. Fernández and Dominique Chapelle (INRIA) for valuable 
discussions about fluid-structure interaction and data assimilation.

\section{References}

[1] S. Balocco, O. Basset, G. Courbebaisse, E. Boni, A.F. Frangi, P. Tortoli, and C. Cachard. Estimation of the viscoelastic properties of vessel walls using a computational model and Doppler ultrasound. Physics in Medicine and Biology, 55(12):3557, 2010.

[2] S. Balocco, O. Basset, G. Courbebaisse, E. Boni, P. Tortoli, and C. Cachard. Noninvasive Young's modulus evaluation of tissues surrounding pulsatile vessels using ultrasound doppler measurement. Ultrasonics, Ferroelectrics and Frequency Control, IEEE Transactions on, 54(6):1265-1271, 2007.

[3] H. T. Banks and K. Kunisch. Estimation techniques for distributed parameter systems, volume 1 of Systems $\&$ Control: Foundations $\&$ Applications. Birkhäuser Boston Inc., Boston, MA, 1989.

[4] D.C. Barber and D.R. Hose. Automatic segmentation of medical images using image registration: diagnostic and simulation applications. Journal of Medical Engineering \& Technology, 29(2):53-63, 2005.

[5] D.C. Barber, E. Oubel, A.F. Frangi, and D.R. Hose. Efficient computational fluid dynamics mesh generation by image registration. Medical Image Analysis, 11(6):648 662, 2007.

[6] D.C. Barber, C. Staicu, I. Valverde, P. Beerbaum, and D.R. Hose. Registration based segment growing for vascular segmentation. Submitted to IEEE Transactions on Medical Imaging, 2012.

[7] C. Bertoglio. Forward and inverse problems in fluid-stucture interaction. Application to hemodynamics. PhD thesis, Universié Pierre et Marie Curie, Paris, France, 2012.

[8] C. Bertoglio, A. Caiazzo, and M.A. Fernández. Fractional-step schemes for the coupling of distributed and lumped models in hemodynamics. SIAM J. Sci. Comp., 35(3):B551B575, 2013.

[9] C. Bertoglio, D. Chapelle, M.A. Fernández, J-F. Gerbeau, and P. Moireau. State observers of a vascular fluid-structure interaction model through measurements in the solid. Comp. Meth. Appl. Mech. Engrg., 256:149-168, 2013.

[10] C. Bertoglio, P. Moireau, and J-F. Gerbeau. Sequential parameter estimation in fluidstructure problems. Application to hemodynamics. Int. J. Numer. Meth. Biomed. Engrg., 28:434-455, 2012.

[11] Chris L. de Korte, Hendrik H. G. Hansen, and Anton F. W. van der Steen. Vascular ultrasound for atherosclerosis imaging. Interface Focus, 1(4):565-575, 2011.

[12] M. D’Elia, L. Mirabella, T. Passerini, M. Perego, M. Piccinelli, C. Vergara, and A. Veneziani. Applications of variational data assimilation in computational hemodynamics. Technical Report TR-2011-002, Emory University, 2011. 
[13] M.A. Fernández and J-F. Gerbeau. Algorithms for fluid-structure interaction problems. In L. Formaggia, A. Quarteroni, and A. Veneziani, editors, Cardiovascular Mathematics. Modeling and simulation of the circulatory system, chapter 9, pages 307-346. Springer Verlag, 2009.

[14] M.A. Fernández, J-F. Gerbeau, and C. Grandmont. A projection algorithm for fluidstructure interaction problems with strong added-mass effect. C. R. Math. Acad. Sci. Paris, 342(4):279-284, 2006.

[15] M.A. Fernández, J.F. Gerbeau, and C. Grandmont. A projection semi-implicit scheme for the coupling of an elastic structure with an incompressible fluid. Int. J. Num. Meth. Engrg., 69(4):794-821, 2007.

[16] Christophe Geuzaine and Jean-Francois Remacle. Gmsh: A 3-d finite element mesh generator with built-in pre- and post-processing facilities. International Journal for Numerical Methods in Engineering, 79(11):1309-1331, 2009.

[17] I. Hoteit, D.-T. Pham, and J. Blum. A simplified reduced order Kalman filtering and application to altimetric data assimilation in Tropical Pacific. Journal of Marine Systems, 36(1-2):101-127, 2002.

[18] S. Julier, J. Uhlmann, and H.F. Durrant-Whyte. A new approach for filtering nonlinear systems. In American Control Conference, pages 1628-1632, 1995.

[19] S. Julier, J. Uhlmann, and H.F. Durrant-Whyte. A new method for the nonlinear transformation of means and covariances in filters and estimators. Automatic Control, IEEE Transactions on, 45(3):477-482, 2000.

[20] P.-Y. Lagrée. An inverse technique to deduce the elasticity of a large artery. Eur. Phys. J. AP, 9:153-163, 1999.

[21] S. Laurent, J. Cockcroft, L. Van Bortel, P. Boutouyrie, C. Giannattasio, D. Hayoz, B. Pannier, C. Vlachopoulos, I. Wilkinson, and H. Struijker-Boudier. Expert consensus document on arterial stiffness: methodological issues and clinical applications. European Heart Journal, 27(21):2588-2605, 2006.

[22] V. Martin, F. Clément, A. Decoene, and J-F. Gerbeau. Parameter identification for a one-dimensional blood flow model. ESAIM-Proceedings, 14:174-200, 2005.

[23] P. Moireau, C. Bertoglio, N. Xiao, C.A. Figueroa, C.A. Taylor, D. Chapelle, and J.-F. Gerbeau. Sequential identification of boundary support parameters in a fluid-structure vascular model using patient image data. Biomechanics and Modeling in Mechanobiology, pages 1-22, 2012.

[24] P. Moireau and D. Chapelle. Reduced-order Unscented Kalman Filtering with application to parameter identification in large-dimensional systems. COCV, 17:380-405, 2011. doi:10.1051/cocv/2010006.

[25] P. Moireau, D. Chapelle, and P. Le Tallec. Filtering for distributed mechanical systems using position measurements: Perspective in medical imaging. Inverse Problems, 25(3):035010-035035, March 2009. 
[26] P. Moireau, N. Xiao, M. Astorino, C.A. Figueroa, D. Chapelle, C.A. Taylor, and JF. Gerbeau. External tissue support and fluid-structure simulation in blood flows. Biomechanics and Modeling in Mechanobiology, 2011.

[27] W.W. Nichols, M.F. O'Rourke, and C. Hartley. McDonald's blood flow in arteries: theoretical, experimental and clinical principles, volume 340809418. Hodder Arnold London, 2005.

[28] J. Peiró and A. Veneziani. Reduced models of the cardiovascular system. In L. Formaggia, A. Quarteroni, and A. Veneziani, editors, Cardiovascular Mathematics. Modeling and simulation of the circulatory system, chapter 10, pages 347-394. Springer Verlag, 2009.

[29] M. Perego, A. Veneziani, and C. Vergara. A variational approach for estimating the compliance of the cardiovascular tissue: An inverse fluid-structure interaction problem. SIAM J. Sc. Comp., 33(3):1181-1211, 2011.

[30] D.-T. Pham, J. Verron, and L. Gourdeau. Filtres de Kalman singuliers évolutifs pour l'assimilation de données en océanographie. Comptes Rendus de l'Académie des Sciences - Series IIA, 326(4):255-260, 1998.

[31] J. Stalhand. Determination of human arterial wall parameters from clinical data. Biomechanics and Modeling in Mechanobiology, 8(2):141-148, 2009.

[32] D.A. Woodrum, A.J. Romano, A. Lerman, U.H. Pandya, D. Brosh, P.J. Rossman, L.O. Lerman, and R.L. Ehman. Vascular wall elasticity measurement by magnetic resonance imaging. Magnetic Resonance in Medicine, 56(3):593-600, 2006.

[33] L. Xu, J. Chen, M. Yin, K.J. Glaser, Q. Chen, D.A. Woodrum, and R.L. Ehman. Assessment of stiffness changes in the ex vivo porcine aortic wall using magnetic resonance elastography. Magnetic Resonance Imaging, 30:122-127, 2012. 